\title{
MUJER, SALUD Y TRADICIÓN EN LOS U'WA DE COLOMBIA Y CUESTIONES DE GÉNERO EN LAS COMUNIDADES INDÍGENAS
}

\author{
ELIZABETH MORA TORRES \\ HAYMETH CECILIA PÁEZ MOLINA, \\ WELLINGTON QUIÑÓNEZ DAZA Y \\ MIGUEL LEONARDO LÓPEZ M. \\ UPTC (Universidad Pedagógica y Tecnológica de Colombia) y \\ Hospital Especial de Cubará (Colombia)
}

\section{INTRODUCCIÓN}

Se conoce muy poco de las comunidades indígenas U'wa de Sudamérica. En particular, de sus condiciones de vida, ritos, métodos de curación y tradiciones. Mostraremos aquí parte de una investigación de campo que trata de remediar estas falencias. Nos centraremos en aspectos relacionados con la salud y comentaremos, en particular, la situación de la mujer U'wa. También nos referiremos, brevemente, a algunos ritos y tradiciones que afectan de manera muy especial a las jóvenes que empiezan su etapa fértil. Veremos que diferentes tradiciones y costumbres impiden, en ocasiones, desarrollar una medicina preventiva tanto en mujeres en edad fértil como en sus bebés. Describiremos también cómo se han tratado las cuestiones de género en diversas comunidades indígenas de América.

En el primer apartado presentaremos la situación sociopolítica de estas comunidades nativas, y definiremos la muestra objeto del estudio. Aportaremos datos sobre la composición familiar, sus costumbres higiénicas y estilo de vida, sus creencias y ritos de purificación, etc. En el apartado siguiente ampliaremos el análisis del sistema de salud indígena y daremos datos sobre la situación actual en términos de enfermedades, vacunaciones, lactancia materna, y nacimientos y muertes. Por falta de espacio no recogeremos aquí con detalle las celebraciones religiosas y prácticas tradicionales de este grupo social. Por otra parte, es muy escasa la bibliografía respecto de estas comunidades indígenas, tanto en formato tradicional como presente en Internet. En nuestra página 
Figura 1: Ubicación geográfica de las comunidades indígenas (áreas oscurecidas y con círculos) en parte de la frontera de Colombia con Venezuela y Brasil. La flecha indica la posición aproximada de la comunidad U'wa objeto de esta investigación, en el departamento de Boyacá. En el extremo inferior izquierdo de la figura se encuentra la capital del país, Bogotá

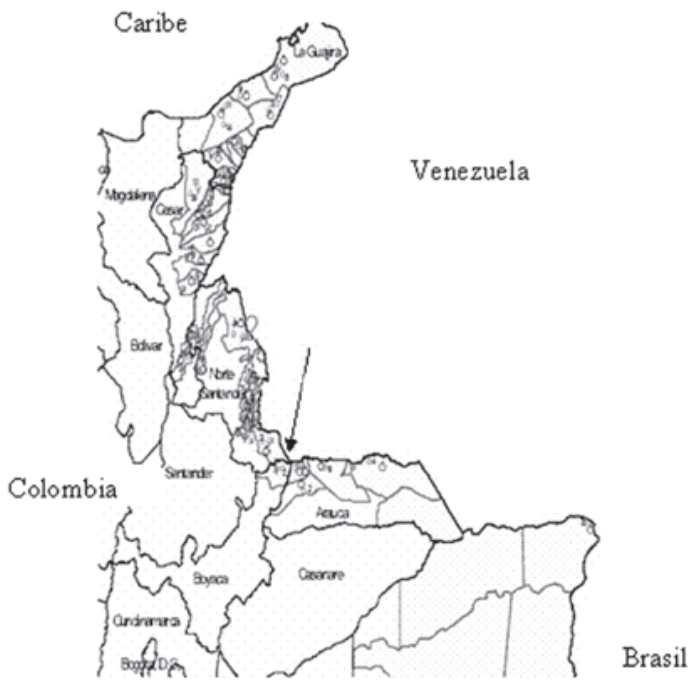

Adaptada de: Inventario de Instituciones y Programas de Salud en las Fronteras Andinas, Bogota, Organismo Andino de Salud, Convenio Hipólito Unanue. http://www.conhu.org.pe/docpasafro/ proyfrontversion1.pdf (consultado el 16 de agosto de 2007).

web ${ }^{1}$, así como en la del Municipio de Cubará2, se pueden consultar más detalles sobre estas prácticas tradicionales. Son de notar, en particular, las tradiciones que afectan a la población femenina, como la relativa a la pubertad de las jóvenes y el ritual de la kókora. Por su interés, haremos una breve reseña de éste en la sección final del trabajo. También analizaremos brevemente algunas cuestiones de género en las comunidades indígenas americanas.

\section{EL PUEBLO U'WA}

Los U'wa son descendientes de los chibchas, pueblos que habitaban las altiplanicies de la cordillera oriental de los Andes, en los departamentos de Cundinamarca y Boyacá (Colombia), hasta su conquista por los españoles en el siglo XVI. El nombre U'wa significa "gente inteligente que sabe hablar». Los

1. MORA TORRES, E.: "Ceremonias religiosas y prácticas sociales del pueblo U'wa (2007)», en http:// emt.saef-ci.com/docs-diversos/Mujer-Uwa-ceremonia-kokora.pdf (consultado el 8 de septiembre de 2007).

2. «Monografía del municipio de Cubará». Gobierno de Cubará, Boyacá, Colombia. http://www. cubara.gov.co/docs/mografia_cubara.doc (consultado el 11 de mayo de 2007). 
Mujer, salud y tradición en los U'wa de Colombia y cuestiones de género en las...

U'wa han sido capaces de mantener firmemente su cultura ancestral hasta bien entrado el siglo XX. Su lengua, U'wajka (o "alma de la gente») pertenece a la macrofamilia lingüística chibcha y tiene tres variantes dialectales: Cobaria, Barronegro y Aguablanca ${ }^{3}$. Según el censo nacional del 2005, en el departamento de Boyacá (el que más alberga a esta comunidad) hay 5776 indígenas U'wa ${ }^{4}$.

El mapa de la fig.1 muestra algunas poblaciones indígenas en una parte de las fronteras orientales de Colombia. También en las fronteras con Perú y Ecuador hay una gran cantidad de comunidades indígenas.

El resguardo indígena U'wa se creó en 1979; se denomina Distrito Fronterizo de Cubará y contiene el municipio de Cubará, que le da el nombre. Con una extensión de $1155 \mathrm{~km}^{2}$, representa el 5\% del total del departamento de Boyacá. El resguardo se encuentra en la vertiente del Orinoquía, al nororiente del departamento, en la frontera natural del río Arauca, límite con la República de Venezuela y a unos $550 \mathrm{~km}$ de Bogotá. La temperatura promedio anual en el resguardo es de $26^{\circ} \mathrm{C}$, con precipitaciones medias anuales de $2920 \mathrm{~mm}$ y a una altura de 420 m.s.n.m. ${ }^{5}$

\subsection{Economía y perfil político administrativo}

La economía de los U'wa es estrictamente de autoconsumo y subsistencia, y generan muy escasos recursos con la venta de productos de artesanía, o con el trabajo asalariado que realizan en el centro urbano, o en veredas cercanas a su comunidad ${ }^{6}$. Los U'wa son recolectores y agricultores: cultivan maíz, plátano, yuca y frijoles; crían pollos y cazan pequeños roedores, así como venado, lapa, y pava, tanto con trampas como con arcos y flechas.

Cada familia tiene dos o tres viviendas, situadas en diferentes niveles en la ladera de la montaña, a donde van a vivir según sea época de siembra o de cosecha. Viven en un clima húmedo, con cambios bruscos de temperatura y tienen limitaciones en la disponibilidad de alimentos. En la actualidad, los indígenas consideran que la exploración y explotación petrolera, así como la tala de los bosques, es la causa principal de la baja producción de sus cosechas y de lo poco nutritivos que resultan los productos agrícolas.

La construcción de las viviendas con estilo propio se ha mantenido a lo largo de los años, considerándose una de las características más tradicionales de esta cultura; se ha conservado el uso de materiales como madera, palma o bijao. La proporción de casas que están construidas con paredes de ladrillo o cemento

3. CHÁVEZ, Á.: Reseña etnográfica de los Cobaria, Pamplona, Casa colonial, 1965.

4. DANE (Departamento Administrativo Nacional de Estadística): XVII Censo Nacional de población y vivienda 2005. Estadísticas de grupos étnicos (2005). http://www.dane.gov.co (consultado el 5 de febrero de 2007).

5. Piñeros-Petersen M, Ruiz Salguero M.: "Aspectos demográficos en comunidades indígenas de tres regiones de Colombia», Salud Pública, 40 (1998), pp. 324-329.

6. Aguablanca, E. B. (Berichá): Tengo los pies en la cabeza, Santa Fe de Bogotá, Los Cuatro Elementos. Banco de la República - Biblioteca Luís Ángel Arango, 1992. (consultado el 12 de octubre de 2006 en http://www.lablaa.org/blaavirtual/modosycostumbres/tengo/indice.htm). 
y techo de zinc o de barro es muy baja, del 4,7\%. En la sección 3.1 se amplían estos datos.

La población indígena se rige por un sistema tradicional de gobierno. La máxima autoridad en cada comunidad es el Werjayá, seguido por un cacique menor (Werjayá menor) y el Cabildo. La representación de las comunidades U'wa se centra en el Cabildo Mayor, cuyo presidente es el representante legal (político y administrativo) de todas las comunidades indígenas ante las instancias nacionales e internacionales.

\subsection{Objetivos del estudio}

De acuerdo con datos oficiales referidos a las comunidades indígenas de Colombia ${ }^{7}$, el perfil epidemiológico presente en las poblaciones indígenas, en general, está asociado a altos índices de pobreza, desempleo, analfabetismo, migración, marginación, falta de tierra y territorio, destrucción del ecosistema, alteración de la dinámica de vida, y necesidades básicas insatisfechas (NBI). Un objetivo del presente trabajo es recoger y analizar datos que confirmen o desmientan estas afirmaciones, para el caso concreto de la población U'wa del Departamento de Boyacá. Por razones de espacio nos centraremos en el análisis del estado de salud de la población. Analizaremos también datos correspondientes a prácticas médicas tradicionales, en comparación con la medicina occidental. Se investigará, en particular, la situación del segmento femenino de la población. Los datos más exhaustivos que se recopilaron en este trabajo se encuentran en la documentación de nuestro hospital ${ }^{8}$.

Por la relación que guardan, en ocasiones, tradiciones y costumbres con prácticas de salud e higiene, se recogen a lo largo del trabajo, y al final del mismo, breves síntesis de creencias y rituales que afectan a esta comunidad, en particular a las mujeres U'wa.

\section{MUESTRA ESTADÍSTICA}

Con el fin de investigar el estado de salud y las costumbres de los indígenas colombianos U'wa se realizó un estudio descriptivo, de corte transversal, de la población indígena U'wa del departamento de Boyacá (Colombia). Se aplicó una encuesta estructurada sobre composición familiar, nivel educativo, afiliación al sistema de seguridad social, condiciones de la vivienda y actividad laboral, además de obtener datos para la caracterización del perfil de morbilidad y mortalidad ${ }^{9}$. Se estableció la muestra con un nivel de confianza del $95 \%$ y el muestreo aleatorio resultó en 362 familias. Los individuos que se rehusaron a

\footnotetext{
7. MPS/OPS: Insumos para la conceptualización y discusión de una Politica de Protección Social en Salud para los Grupos Étnicos en Colombia, Bogotá, Informes del Ministerio de la Protección Social, OPS (Organización Panamericana de la Salud) y OMS (Organización Mundial de la Salud), 2004.

8. Situación de salud de la comunidad indígena U'wa del Departamento de Boyacá, Colombia, Hospital Especial de Cubará, Gobernación de Boyacá y Secretaría de Salud de Boyacá, Colombia, 2007.

9. Situación de salud de la comunidad indígena U'wa del Departamento de Boyacá, Colombia: Op. cit.
} 
Mujer, salud y tradición en los U'wa de Colombia y cuestiones de género en las...

participar del estudio fueron excluidos. Con ello, el número de familias participantes quedó establecido en 342 y un total de 1353 personas.

En lo referente a la construcción y validación de contenidos, constructo y confiabilidad de la prueba, se realizó un pilotaje a indígenas de una de las comunidades investigadas, que fue ejecutado por el equipo responsable del estudio, con la colaboración de promotores indígenas y de la asociación de autoridades tradicionales y cabildos Asou'wa.

\subsection{Estadística poblacional}

En este apartado se recogen datos sobre composición familiar, nivel educativo y actividad laboral, manejo de basuras, excretas y agua de consumo, tipo de vivienda y alimentación. Los datos más específicos referentes a la salud se presentan más adelante.

Composición familiar

La muestra la componen familias de diez comunidades: Barrosa, Bocotá, Zulia, etc. La edad promedio de los integrantes de la muestra fue de 20 años (tabla 1). Se observa un predominio del sexo femenino, especialmente en algunas comunidades, obteniéndose una razón mujer/hombre de 1,13/1, con un total de 296 hombres como jefes de familia y de 43 mujeres como jefes de familia. El promedio de habitantes por familia es de 4.

Según los datos de la tabla 1 la mayoría de la muestra se ubicó en las categorías «soltero» y "casado», para ambos sexos. Las categorías con más bajo porcentaje fueron, también para ambos sexos, viudo y separado o divorciado. Al comparar los datos correspondientes a hombres y mujeres se aprecia una población muy joven y gran disparidad (con predominio femenino) en los porcentajes de viudedad y separaciones o divorcios. Predominan las mujeres y los hombres casados frente a solteros, aunque la diferencia en los porcentajes de personas casadas respecto de solteras es mucho mayor en el universo femenino (12,6 puntos) que en el masculino (4,7 puntos).

Tabla 1: Distribución de la muestra en función del sexo, edad media y estado civil

\begin{tabular}{|c|c|c|c|c|c|c|c|c|}
\hline Sexo & Núm. & Edad & \multicolumn{7}{|c|}{ Estado civil } \\
\cline { 4 - 8 } & $(\%)$ & media & $\mathbf{S}$ & $\mathbf{C}$ & U. L. & V & S/D & Total \\
\hline Masculino & 638 & 20,7 & 318 & 288 & 25 & 5 & 4 & 640 \\
& $(47,2 \%)$ & & $(49,7 \%)$ & $(45 \%)$ & $(3,9 \%)$ & $(0,8 \%)$ & $(0,6 \%)$ & $(100 \%)$ \\
\hline Femenino & 715 & 19,7 & 371 & 281 & 25 & 19 & 17 & 713 \\
& $(52,8 \%)$ & & $(52 \%)$ & $(39,4 \%)$ & $(3,5 \%)$ & $(2,7 \%)$ & $(2,4 \%)$ & $(100 \%)$ \\
\hline
\end{tabular}

S: soltero/a; C: casado/a; V: viudo/a; U.L: unión libre; S/D: Separado/a o divorciado/a.

Nivel educativo y actividad laboral

La proporción de analfabetismo en la población es muy elevado, del 86,8\%, y con claro predominio de analfabetismo femenino. El 13,2\% cursó algún grado 
en primaria $(12,3 \%)$ o en secundaria $(0,9 \%)$; no existen reportes de estudios superiores tales como técnicos o universitarios.

En relación al trabajo desempeñado, en hombres predomina como principal actividad laboral la agricultura, con un $96.5 \%$, seguido de actividades como la caza y la pesca; en las mujeres sobresale la agricultura, con el 74,6\%, acompañada del cuidado de hijos y la cría de animales. Cabe resaltar que con ninguno de estos trabajos se superan ingresos del orden del salario mínimo legal $(\$ 433.700$, unos $160 € /$ mes, en 2007).

\section{Manejo de basuras, excretas y agua de consumo}

Un $72,7 \%$ de viviendas obtienen el agua de las quebradas, sin tratar. Las demás familias la toman de pozos o tanques $(21 \%)$ y muy pocas viviendas indígenas $(2 \%)$ cuentan con conexión domiciliaria a las fuentes de agua, a través de mangueras.

Un 98,2\% de las familias eliminan las basuras arrojándolas a campo abierto, como abono para las tierras cercanas a sus casas. El 69,9\% de las familias utilizan como servicio sanitario también el campo abierto; el restante, aunque no tiene sanitario ni letrina, cuentan con un lugar especifico.

\section{Tipo de vivienda}

Prevalecen las viviendas tradicionales, úbajas $(95,3 \%)$, construidas principalmente por techos en paja y palma $(81,6 \%)$, paredes de madera $(57,8 \%)$ y el restante de materiales como tejas de barro o zinc y pisos de tierra $(97,7 \%)$. En Zulia y Cobaria algunas familias viven en las antiguas misiones. No se cuenta con iluminación adecuada (se utiliza la hoguera como alumbrado en un $96,5 \%$ ) y existe poca ventilación. Hay presencia de roedores en el 98,2\% de las viviendas. Habitan de 2 a 5 personas por vivienda y crían animales (pollos, perros, cerdos y animales silvestres).

\section{Alimentación}

Dentro de la población estudiada se halló que los principales grupos alimenticios que se consumen son los correspondientes a hortalizas $(95,4 \%)$, frutas $(94,7 \%)$, lácteos $(14,6 \%)$ y granos $(10,5 \%)$, con un promedio de dos comidas al día. De la muestra, el 88,1\% no se encuentra actualmente en programas de ayuda nutricional; el restante recibe ayudas de alimentación escolar, desayunos infantiles y "familias en acción».

La valoración talla/peso indicó que un $97,5 \%$ de la muestra tiene peso adecuado, un 2,5\% (niños y niñas de 0 a 10 años) posee bajo peso; no existen registros de sobrepeso.

En cuanto a consumo de alcohol y sustancias psicoactivas, un $49,1 \%$ de hombres frecuenta embriagarse de dos a tres días a la semana, y generalmente consumen chicha, coca, tabaco mascado y tabaco fumado. En las mujeres, un $47 \%$ frecuenta embriagarse con diversa regularidad; el consumo más alto es de chicha y coca. Como se ve, hay poca diferencia en el consumo de alcohol entre 
Mujer, salud y tradición en los U'wa de Colombia y cuestiones de género en las...

los dos sexos. Este es un reflejo de la situación socioeconómica de la comunidad U'wa.

\section{SALUD E HIGIENE}

El trabajo de campo ha permitido obtener datos cuantitativos y también conocimientos sobre prácticas y creencias de la población. En este apartado presentaremos una descripción general de las costumbres higiénicas, del sistema tradicional de salud indígena, y de cómo se relaciona con el sistema occidental. Comentaremos los factores que condicionan la salud de las comunidades, en particular de las mujeres U'wa, que tienen muchas veces origen en costumbres (que no les permiten, por ejemplo, aceptar exploraciones ginecológicas). En el apartado siguiente daremos y discutiremos algunos datos cuantitativos.

\subsection{Costumbres higiénicas}

Los U'wa tradicionalmente creen que toda persona u objeto foráneo trae contaminación y debe ser purificado. Esto se hace durante cuatro o más noches de cantos, rituales y con soplos sobre una pluma de garza. Antes de 1982 no aceptaban libros, radios o grabadoras y se oponían a cualquier sistema escolar.

Los indígenas que viven dentro de las comunidades hacen lavados de manos y boca antes de comer, y tienen su propia valoración sobre los contaminantes del agua y la comida; en particular, se cuidan de no consumir alimentos ni beber agua en cualquier parte. Tienen una alimentación propia, como su propia concepción sobre el manejo de las basuras, y siguen las normas del Werjayá (autoridad tradicional), quien también es el más sabio agente de salud, y del Tayota (adivino).

\subsection{El sistema de salud tradicional indígena}

El sistema de salud del pueblo U'wa está basado en la tradición y el aprendizaje de diversos mundos (conocimientos de origen), como verdades que sustentan la concepción del origen del mundo, de lo que deben hacer los U'wa por el mundo y para agradar a Sira (Dios o Creador del mundo U'wa). Estos conocimientos dan cuenta de la salud y la enfermedad, sobre la vida y la muerte, y en ellos se cimienta el sistema de medicina tradicional.

La medicina tradicional indígena es orientada y practicada por los Werjayá, que tienen una jerarquía basada en la profundidad de sus conocimientos y en el control que ejercen sobre diferentes fuerzas de la naturaleza y sobrenaturales.

Los Werjayá tienen ayudantes y aprendices, como los Baukarinas y Kuerikukinas. Los Werjaina ubican la salud como un estado de armonía del hombre con la tierra, el aire, el agua, el fuego, los alimentos, y las personas. Los U'wa tienen salud cuando viven con alegría y comen bien. Las prácticas de medicina tradicional se basan en una actividad conocida como el soplo, técnicas de manejo de la energía, el uso de plantas especiales, rezos, ayunos o dietas (libres de sal y de carne, comida cruda, etc.). La mayoría de las familias conocen el uso de plantas medicinales (medicina tradicional). 
Para el U'wa tener salud es estar bien, no tener problemas ni sufrir ninguna enfermedad. Antiguamente la gente se enfermaba poco: no había contaminación del aire ni había quien propagara las enfermedades. Los ancianos Uejená controlaban y soplaban toda clase de objetos traídos de otra región, y lo mismo hacían a las personas que salían de ella a comerciar. A pesar de ello, de vez en cuando llegaba la viruela, el sarampión o la tos ferina, acabando con mucha gente. Las enfermedades pueden ser transmitidas por diversos elementos de la naturaleza como el sol, los espíritus de los muertos, el agua lluvia, la atmósfera, la tierra, el medio ambiente, la rascadera, y algunas fieras y animales, entre otros. Con el propósito de combatir las enfermedades los ancianos Uejená recibieron de Sira distintas fórmulas de curación referidas a cada enfermedad, las cuales se le aplican al paciente mediante recitativos cantados o rezados mentalmente. Si el anciano llega a aplicar la fórmula inadecuadamente es posible que el paciente se complique, se agrave y sobrevenga la muerte.

A pesar del proceso de aculturación al que se han visto sometidos los U'wa por la intromisión religiosa y la presencia colonizadora, las comunidades siguen practicando la medicina tradicional en cabeza de los Werjayá. Sin embargo, ellos admiten que no todas las enfermedades se pueden curar a través de la medicina tradicional y por ello recurren en segunda instancia a la medicina occidental (puestos de salud, hospitales). Así, los enfermos consultan o mandan consultar al Tayota para encontrar el origen de la enfermedad y para saber quién puede curarla. Algunas veces el adivino descubre que se tata de una enfermedad contagiada de blanco y que sólo los blancos saben curar; en ese caso los Werjayá autorizan la salida de los enfermos hacia el centro de salud o el hospital local, si consideran que el cuerpo del indígena está acostumbrado a los medicamentos. En algunas comunidades, el enfermo es visto primero por el médico occidental y al regresar a su comunidad participa de un rito de purificación. Tradicionalmente, si en alguna familia hay un enfermo, toda la familia suele acompañarlo, porque se tiene una concepción grupal de la enfermedad.

Algunas familias tienen su propio método de planificación familiar, y los partos son atendidos por familiares cercanos o por el cacique; las mujeres lactan a sus hijos hasta más o menos los dos años de edad.

\subsection{Factores condicionantes de la salud}

En general, la comunidad indígena tiene un bajo nivel socioeconómico y los recién nacidos tienen bajo peso al nacer debido a los ayunos de las mujeres gestantes, al consumo de hayo (hojas de coca) que hace desaparecer el hambre, al consumo de alimentos poco nutritivos y comidas no equilibradas.

En cuanto a actividades de protección específica, algunas familias rechazan la inmunización vía parenteral en menores de 1 año, cuando el niño o niña aún no ha recibido bautizo y nombre mediante una ceremonia tradicional. Otras familias rechazan la vacunación a cualquier edad. Las actividades como citologías, atención en planificación familiar y atención del parto, no son recibidas por la población por razones culturales. 
Mujer, salud y tradición en los U'wa de Colombia y cuestiones de género en las...

Los U'wa tienen acceso a los diferentes sistemas de salud, tanto de medicina tradicional como el sistema colono. Los «promotores de salud» cumplen el papel de ser intermediarios entre los dos sistemas; son personas ligadas a organizaciones estatales, y algunos a veces son Kuerikukinas (ayudantes del Werjaina), que se encargan principalmente de mantener el contacto entre la medicina de los colonos y la de la población indígena. La ley 100 de 1993 estableció el Sistema General de Seguridad Social e incluyó el derecho de la población indígena a participar de Régimen Subsidiado en Salud. En el presente estudio se encontró que un $83,6 \%$ de la población pertenecen a este régimen, que contrata con instituciones de salud de primer nivel. A estas organizaciones les corresponde, por ley, propiciar diálogos entre los sistemas de salud y la comunidad, para adecuar los servicios asistenciales a las particularidades culturales y sociales de cada región.

En lo concerniente a la atención primaria en salud, se cuenta con las brigadas correspondientes a las Instituciones Prestadoras de servicios de Salud (IPS) de los municipios de Cubará y Güican. Estas brigadas realizan visitas dos veces al año, la primera antes del primer ayuno de kuezko y la segunda en los meses de octubre a noviembre. Estas brigadas cuentan con médico, odontólogo o higienista oral, enfermero y vacunador. Aunque las comunidades cuentan, como se ha dicho, con algunos promotores de salud indígenas capacitados para ejercer una labor de medicina preventiva y de promoción de la salud, debido al difícil acceso geográfico desde las comunidades hacia el hospital algunas personas sufren enfermedades y muertes evitables hoy en día.

\section{PERFIL EPIDEMIOLÓGICO: DATOS ESTADÍSTICOS}

El análisis de la situación en cuanto a salud de la comunidad indígena U'wa precisa de la caracterización de los factores de riesgo presentes y predominantes en esta población. Dado que la salud es un derecho de toda la población, y es el principal indicador de la calidad de vida de las personas ${ }^{10}$, se debe también estimar la cobertura y el acceso a los sistemas de salud en las comunidades indígenas e identificar las barreras que encuentran esta población para acceder a los servicios de salud.

Con objeto de formar un cuadro general sobre la morbilidad y mortalidad del pueblo U'wa, así como de su actitud ante las prácticas y opciones de salud que son accesibles al resto de la población colombiana, presentaremos a continuación un conjunto de datos estadísticos tomados de la muestra referida en la sección 3. Al concluir esta batería de datos presentaremos, en el apartado siguiente, unas reflexiones generales y una comparación de la situación sanitaria según el sexo y la edad.

10. IbacAche, J; Leighton, A.: Perfil epidemiológico de los pueblos indígenas, Informe del Ministerio de Salud de Colombia, 1999. http://www.ministeriodesalud.go.cr/ops/documentos/ (consultado el 23 de abril de 2007). 


\section{Morbilidad y mortalidad}

A partir de los datos de las instituciones prestadoras de salud, los motivos de consulta que ocuparon las cinco primeras causas de atención en el año 2006 fueron la bronquitis $(27 \%)$, la acidez gástrica $(25 \%)$, la enfermedad diarreica aguda (19\%), la infección respiratoria aguda (15\%) y la parasitosis $(10 \%)$. No se tienen datos referentes a la atención de partos, porque mayoritariamente ocurren en las propias viviendas y por el sistema tradicional. Los cambios en el estado del tiempo, la contaminación ambiental, la caída de cuajo, la herencia y el shaguara (mal de ojo) son las principales causas que atribuyeron las personas encuestadas a las anteriores enfermedades.

Los datos de morbilidad sentida ${ }^{11}$ se recogen en la tabla 2 , y coinciden con los datos de las Instituciones Prestadoras de servicios de Salud (IPS). Se observa que la población masculina es la que con menor frecuencia asiste a los servicios de salud, y bebés y niñas son las más atendidas.

Tabla 2: Distribución de la muestra por morbilidad sentida y grupos más afectados

\begin{tabular}{|l|c|c|c|c|}
\hline \multirow{2}{*}{ Enfermedad } & Frecuencia & \multirow{2}{*}{$\%$} & \multicolumn{2}{c|}{$\begin{array}{c}\text { Grupo poblacional más afectado } \\
\text { por sexo y rango de edad }\end{array}$} \\
\cline { 3 - 5 } & & & Sexo & Años \\
\hline Diarrea & 145 & $35.9 \%$ & $\mathrm{~F}$ & $0-10$ \\
\hline Tos & 117 & $29 \%$ & $\mathrm{~F}$ & $0-10$ \\
\hline Fiebre & 116 & $27 \%$ & $\mathrm{~F}$ & $0-10$ \\
\hline Gripe & 90 & $22,7 \%$ & $\mathrm{~F}$ & $0-10$ \\
\hline Dolor de cabeza & 77 & $18 \%$ & $\mathrm{~F}$ & $21-30$ \\
\hline Dolor en el pecho & 65 & $13,7 \%$ & $\mathrm{~F}$ & $11-30$ \\
\hline Dolor de estomago & 48 & $10,1 \%$ & $\mathrm{~F}$ & $0-10$ \\
\hline Dolor de espalda & 32 & $7,3 \%$ & $\mathrm{M}$ & $41-50$ \\
\hline Dolor en el cuerpo & 31 & $6,8 \%$ & $\mathrm{~F}$ & $21-60$ \\
\hline Granos en el cuerpo & 20 & $4,1 \%$ & $\mathrm{M}$ & $0-10$ \\
\hline Escalofríos & 20 & $3,9 \%$ & $\mathrm{~F}$ & $21-30$ \\
\hline Dolor de cintura & 14 & $3,7 \%$ & $\mathrm{M}$ & $31-40$ \\
\hline Total & 775 & $100 \%$ & & \\
\hline
\end{tabular}

Con relación a qué servicios acude la población enferma, se observa en la fig. 2 que un $39,4 \%$ acude a atención en el nivel 1 y el 16,7\% asiste con el promotor de salud u otro familiar; con el médico tradicional se totaliza un $9,6 \%$ y una proporción del $34,3 \%$ opta porque nadie trate su enfermedad. Por tanto, un poco más de una cuarta parte de las personas encuestadas acuden a los servicios tradicionales o promotores indígenas $(16,7 \%+9,6 \%)$. Este hecho es una muestra del rápido proceso de introducción de la medicina occidental en estas comunidades, ocurrido en poco más de dos decenios.

11. "Sentida» se refiere a la opinión (no médica) de familiares, comunidad, etc. También se denomina "estado de salud autopercibido». 
Mujer, salud y tradición en los U'wa de Colombia y cuestiones de género en las...

Figura 2: Distribución de la población por servicios de salud consultados

Nadie Nivel I de atención

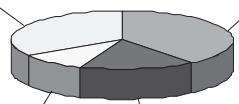

Médico tradicional

Promotor o familiar

Durante el año 2006 y los cuatro primeros meses del año 2007 se registraron 51 fallecimientos (tabla 3); hubo un predominio del sexo masculino, con un $64 \%$ de fallecimientos. El promedio de edad en el momento de la muerte fue de 28 años, pero entre el rango de 0 a 10 años se presentó el mayor porcentaje de defunciones. En este periodo de tiempo no se reportaron muertes maternas durante el parto.

Tabla 3: Indicadores de mortalidad sentida en todos los grupos de edad

\begin{tabular}{|l|c|c|c|}
\hline \multicolumn{1}{|c|}{ Causa de la muerte } & Frecuencia & \multicolumn{2}{c|}{$\begin{array}{c}\text { Grupo poblacional } \\
\text { más afectado }\end{array}$} \\
\cline { 3 - 4 } & & Sexo & Rango edad \\
\hline Infección respiratoria aguda & 14 & $\mathrm{M}$ & $0-10$ \\
\hline Vejez & 9 & $\mathrm{M}$ & $61-70$ \\
\hline Enfermedad diarreica aguda & 6 & $\mathrm{~F}$ & $0-10$ \\
\hline Mordedura de serpiente & 5 & $\mathrm{M}$ & $21-30$ \\
\hline Desconocida & 5 & $\mathrm{M}$ & $61-70$ \\
\hline Enfermedad pulmonar & 3 & $\mathrm{~F}$ & $0-10$ \\
\hline Tuberculosis & 2 & $\mathrm{M}$ & $41-60$ \\
\hline Ahogo & 2 & $\mathrm{M}$ & $51-60$ \\
\hline Paludismo & 1 & $\mathrm{M}$ & $0-10$ \\
\hline Otras causas & 4 & $\mathrm{M}$ & $11-20$ \\
\hline Total & 51 & & \\
\hline
\end{tabular}

Vacunación, salud oral, desparasitación y lactancia materna en menores de cinco años

De acuerdo a los registros de vacunación, tabla 4, un 14,5\% de niños indígenas U'wa no han sido vacunados en ninguna ocasión. Los principales motivos que se reportaron para la ausencia de vacunación fueron los siguientes: por vivir muy lejos del centro de salud $(44,4 \%)$, porque no ha sido bautizado el niño o la niña $(33,3 \%)$ y por no querer vacunarlo los padres $(19,4 \%)$. Sin embargo, dentro del porcentaje de niños que sí que han sido vacunados la mayoría no presenta el esquema de vacunación adecuado para su edad. Los datos concretos varían fuertemente de comunidad en comunidad. Estos datos de vacunación, así como los hábitos alimenticios ya comentados, permiten entender los elevados índices de mortalidad infantil que muestra la tabla 3. 
Elizabeth Mora Torres, Haymeth Cecilia Páez Molina, Wellington Quiñónez Daza y Miguel...

Tabla 4: Menores de cinco años vacunados según un esquema adecuado, o no, en una distribución de la muestra por comunidades

\begin{tabular}{|l|c|c|c|c|}
\hline \multirow{2}{*}{ Comunidad } & \multicolumn{2}{|c|}{ Vacunados } & \multicolumn{2}{c|}{ Esquema adecuado } \\
\cline { 2 - 5 } & Sí & No & Sí & No \\
\hline Barrosa & 25 & 0 & 10 & 15 \\
\hline Bocotá & 12 & 2 & 0 & 14 \\
\hline Cobaria & 35 & 6 & 8 & 33 \\
\hline Chuscal & 12 & 3 & 5 & 10 \\
\hline Fátima & 20 & 2 & 11 & 11 \\
\hline Rabaria & 17 & 9 & 3 & 23 \\
\hline Rinconada & 8 & 4 & 1 & 11 \\
\hline Rotarbaria & 24 & 4 & 10 & 18 \\
\hline Tegria & 35 & 3 & 21 & 17 \\
\hline Zulia & 48 & 7 & 32 & 23 \\
\hline Total & 236 & 40 & 101 & 175 \\
\hline
\end{tabular}

Normalmente, la población U'wa no tiene alteraciones dentales. En referencia a la consulta odontológica en el último año (2006-2007) se halló que un $97,1 \%$ no asiste por desconocimiento, lejanía u otras causas. Mas de la mitad de esta muestra no tiene caries, y la proporción de menores con caries es muy baja, del 2,2\%.

El porcentaje de desparasitados es de $68,6 \%$, donde prima una sola desparasitación en el último año, con un 92,6\%. El total de desparasitados fue de 102 menores, un $37 \%$.

En cuanto a hábitos de lactancia, se destaca (tabla 5) que sólo un/a niño/a nunca recibió lactancia materna y un $44,4 \%$ la tomó hasta el segundo año de

Tabla 5: Lactancia materna en menores de cinco años en una distribución de la muestra por comunidades

\begin{tabular}{|l|c|c|c|c|c|c|c|}
\hline \multirow{2}{*}{ Comunidad } & \multicolumn{4}{|c|}{ Lactancia } & \multicolumn{4}{c|}{ Lactó hasta... } \\
\cline { 2 - 8 } & Ex. & Com. & N.L. & $\begin{array}{c}\mathbf{6} \text { meses } \\
\text { 1 año }\end{array}$ & $\begin{array}{c}\mathbf{1} \text { a 2 } \\
\text { años }\end{array}$ & $\begin{array}{c}\text { Más de 2 } \\
\text { años }\end{array}$ & $\begin{array}{c}\text { Nunca } \\
\text { recibió }\end{array}$ \\
\hline Barrosa & 7 & 7 & 11 & 5 & 6 & 0 & 0 \\
\hline Bocotá & 4 & 6 & 4 & 1 & 3 & 0 & 0 \\
\hline Cobaria & 5 & 14 & 22 & 12 & 9 & 1 & 0 \\
\hline Chuscal & 7 & 4 & 4 & 2 & 2 & 0 & 0 \\
\hline Fátima & 2 & 8 & 12 & 6 & 4 & 2 & 0 \\
\hline Rabaria & 6 & 8 & 12 & 5 & 7 & 0 & 0 \\
\hline Rinconada & 2 & 5 & 5 & 2 & 3 & 0 & 0 \\
\hline Rotarbaria & 5 & 13 & 10 & 7 & 3 & 0 & 0 \\
\hline Tegria & 3 & 16 & 19 & 5 & 12 & 2 & 0 \\
\hline Zulia & 12 & 18 & 25 & 17 & 6 & 1 & 1 \\
\hline Total & 53 & 99 & 124 & 62 & 55 & 6 & 1 \\
\hline
\end{tabular}

Tipo de lactancia: Ex.: exclusiva, Com.: complementaria, N.L.: no está lactando. 
Mujer, salud y tradición en los U'wa de Colombia y cuestiones de género en las...

edad. Sin embargo, la lactancia materna en exclusiva es poco frecuente, probablemente por las deficiencias dietéticas de las madres.

\section{Actividades de promoción y prevención en mujeres en edad fértil}

En la muestra se encontró a 404 mujeres (33,4\%) en edad fértil, con una edad de inicio de la maternidad de 14 años. Dentro de esta población femenina sólo una mujer planifica, utilizando el método tradicional cuhwiro o soplo, que es realizado por el cacique de la comunidad. Los siguientes motivos se expresaron para no planificar: no desearlo, creencias culturales o no tener actualmente compañero. Ninguna de las mujeres encuestadas se practicó examen de matriz en el último año, principalmente debido a las creencias culturales y al desconocimiento. Por las mismas razones, ninguna mujer en edad fértil se realizó examen de seno en el último año. Vemos que las mujeres U'wa comienzan a tener hijos cuando son muy jóvenes $y$, por otra parte, les resulta difícil someterse a controles ginecológicos.

\section{Gestación, embarazo y posparto}

Dentro de la población femenina que se hallaba en edad fértil se encontraban 58 mujeres (14,5\%) en estado de embarazo; de este grupo, sólo un 12,1\% ha asistido a controles, ya sea con las instituciones de salud o con el promotor de salud; las demás no utilizan este servicio por lejanía, dificultades económicas o desconocimiento. A un $65,1 \%$ de mujeres embarazadas se les ha vacunado con la primera dosis tetánica.

Según los datos de la tabla 6, la mayor fracción de mujeres ha tenido de 3 a 5 hijos, y a casi una cuarta parte de ellas se les ha muerto algún hijo al nacer. Tradicionalmente, los partos han sido asistidos por ellas mismas y en algunos casos recurren a familiares o parteras; actualmente, una baja proporción acude a ser tratada por los sistemas de salud.

Tabla 6: Distribución de la muestra por nacidos vivos y nacidos muertos.

\begin{tabular}{|l|c|c|c|c|c|}
\hline $\begin{array}{c}\text { Nacidos } \\
\text { vivos }\end{array}$ & Frecuencia & $\%$ & $\begin{array}{c}\text { Nacidos } \\
\text { muertos }\end{array}$ & Frecuencia & $\%$ \\
\hline $1-2$ & 124 & $38,4 \%$ & $1-2$ & 77 & $23,9 \%$ \\
\hline $3-5$ & 154 & $47,7 \%$ & $3-5$ & 4 & $1,2 \%$ \\
\hline $5-7$ & 35 & $10,8 \%$ & $5-7$ & 1 & $0,3 \%$ \\
\hline 8 o más & 3 & $0,9 \%$ & 8 o más & 0 & $0 \%$ \\
\hline Ninguno & 7 & $2,2 \%$ & Ninguno & 241 & $74,5 \%$ \\
\hline Total & 323 & $100 \%$ & Total & 323 & $100 \%$ \\
\hline
\end{tabular}

Entre las enfermedades que con mayor frecuencia han afectado a las mujeres en el embarazo anterior, o en el actual, se encontraron, por este orden: hemorragias, infección urinaria, anemia, y diabetes, figura 3.

Aunque el estudio estadístico realizado fue mucho más exhaustivo que el presentado aquí, con estos datos podemos tener una idea cabal de la situación 
Figura 3: Distribución de la muestra por enfermedades durante el anterior o actual embarazo

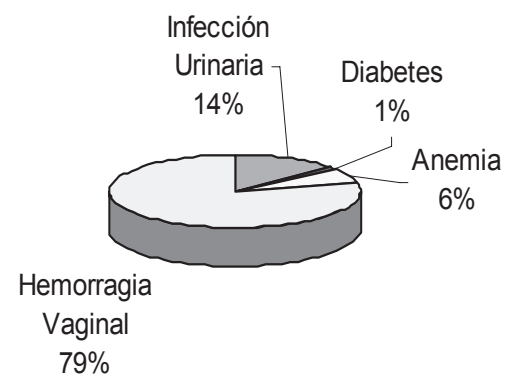

higiénico-sanitaria de este grupo poblacional, y el análisis de algunas de sus tradiciones y hábitos nos ha permitido contextualizar estos datos. Presentaremos a continuación algunos comentarios generales en este sentido.

\section{ANÁLISIS DEL ESTUDIO DE CAMPO Y DISCUSIÓN}

Los datos mostrados en la sección anterior indican que en lo concerniente a la composición familiar de las comunidades indígenas U'wa predomina la presencia de mujeres, en especial en el rango de edad de 0 a 10 años. Se produce una gran movilización de los hombres por motivos de trabajo en la agricultura $y$, además, son ellos quienes más asisten a las escuelas. En cuanto al nivel educativo, se observó una alta proporción de analfabetismo, especialmente en las mujeres U'wa. Este hecho, sumado a la baja compensación económica, ubica a esta comunidad como de alta dependencia económica frente a la nación ${ }^{12}$.

Las creencias culturales de la comunidad U'wa los lleva a seleccionar las quebradas o pozos de donde es posible beber el agua sin temor a enfermarse, ya que consideran a los ríos fuentes de aguas contaminadas natural y espiritualmente. Pese a esta concepción de pureza, el consumo de agua no potable es una de las principales causas de morbilidad, especialmente de parasitosis en niñas y niños menores de cinco años.

Las cifras de desnutrición se centran en menores de 10 años; en especial, los recién nacidos tienen bajo peso al nacer debido a los ayunos de las madres, al consumo de alimentos poco nutritivos, comidas no balanceadas y el consumo de hojas de coca para mitigar la necesidad de comer. Debido a ello, los miembros de estas comunidades tienen pocas defensas en sus organismos para combatir graves enfermedades que ponen en peligro su vida. Estos resultados se asemejan a los encontrados en las comunidades Paeces en el Cauca, Awá

12. SÁnChez, E; ARAngo, R.: Los Pueblos indigenas de Colombia en el umbral del nuevo milenio, Bogotá, Departamento Nacional de Planeación, 2004. 
Mujer, salud y tradición en los U'wa de Colombia y cuestiones de género en las...

Kwaikeres en Nariño y Emberá en Antioquia, en donde una de las principales causas de mortalidad infantil es la desnutrición ${ }^{13}$.

Los datos de vacunación mostraron una creciente incidencia de los sistemas de salud dentro de la cultura U'wa. Pese a ello, la distancia existente entre las comunidades y las instituciones de salud se consolida como el primer obstáculo para la inmunización por vía oral o parenteral; este hecho desplaza en importancia a las creencias culturales, tales como el no estar aún bautizado y no contar, por tanto, con el nombre U'wa, como principales motivos que años atrás impedían realizar este tipo de actividades de promoción y prevención.

El desconocimiento, la lejanía, además de sus costumbres en relación al lavado de boca y dientes mediante el uso de plantas, hacen concebir que no sea necesario asistir al odontólogo; no es ésta una necesidad básica dentro de su cultura de bienestar y salud.

La edad de inicio de la actividad sexual encontrada en mujeres embarazadas, o con su primer hijo, fue de 14 años en adelante y se mantiene hasta aproximadamente los 45 años. Respecto a las actividades de promoción y prevención, un alto porcentaje de la población femenina adulta no planifica, no se realiza citologías, examen de seno, ni controles prenatales. Muchas mujeres opinan que planificar interrumpe el ciclo de vida de los U'wa, donde la fertilidad es un estado muy valioso para la mujer y para su cultura. El impedir que una mujer procree es una negación hacia la prosperidad y trascendencia en el tiempo de sus comunidades.

En los U'wa la morbilidad, tanto sentida como institucional, mostró que la enfermedad diarreica aguda, infección respiratoria aguda y parasitosis fueron las que prevalecieron. De acuerdo con el Ministerio de Protección Social ${ }^{14}$ y la OPS/OMS ${ }^{15}$, éstas son enfermedades propias de la pobreza y situaciones de marginación.

En la actualidad se ha modificado la percepción sobre los sistemas de salud, haciéndose más cercanos y efectivos en la población indígena, e incrementando paulatinamente el acceso a estos servicios. Pese a que el médico tradicional posee una sabiduría basada en el conocimiento profundo de su medio, sus relaciones, recursos y aplicabilidad, y muestra un gran nivel de efectividad en la curación de dolencias y enfermedades propias de su entorno, ciertas enfermedades ajenas a su cultura requieren de otro tipo de tratamiento. Características tan propias y arraigadas de una cultura, como son el idioma y las creencias culturales, no representan un obstáculo actualmente para acceder a los servicios

13. AUPEC (Agencia Universitaria de Periodismo Científico): Población indígena, en riesgo de desaparecer, 2000. http://aupec.univalle.edu.co/temas/civilizacion.html (consultado el 25 de abril de 2007).

14. MPS/OMS: Op. cit.

15. OPS/OMS: Iniciativa de salud de los pueblos indígenas. División de Desarrollo de sistemas y servicios de salud. División de promoción y protección de la salud. Salud Mental y poblaciones indígenas en América Latina, Ginebra, Organización Panamericana de la Salud / Organización Mundial de la Salud, 1997. 
en salud de las IPS, que cada vez convocan a un número mayor de indígenas de todas las edades.

Entre los factores de riesgo que potencializan las enfermedades en esta población se hallaron los siguientes: cambios bruscos de temperatura, consumo de agua no potable, inadecuada alimentación y atención médica tardía. Estos factores se asemejan a los encontrados en las comunidades indígenas a orillas del rió Putumayo en $1998^{16}$. El no contar con registros reales y actualizados de mortalidad dificulta las actividades de los sistemas de salud, ya que algunas personas sufren enfermedades y muertes evitables hoy en día. Cabe resaltar, sin embargo, que no existen reportes de fallecimientos por actos de violencia, lo que define un sistema de protección social en estas comunidades indígenas del Departamento de Boyacá

El presente estudio ofrece una visión más clara y complementaria de la situación de salud de los indígenas U'wa, y permite también caracterizar sus condiciones de vida de acuerdo con su contexto cultural. Muchos de los resultados del estudio coinciden con otros similares ${ }^{17}$, y refuerzan la necesidad de una mayor atención a estas comunidades, especialmente a las mujeres adultas en edad fértil y a sus bebés.

\section{RITOS Y TRADICIONES}

Ya se ha comentado cómo diversas creencias y tradiciones impiden el acceso a prácticas de salud (como vacunaciones en bebés o exploraciones preventivas en mujeres). Mencionaremos brevemente aquí un ritual importante en las jóvenes de estas comunidades indígenas, el de la imposición y posterior quitada de la kókora o gorro que les cubre la cabeza:

«La mujer queda incomunicada desde el instante en que le llega la primera menstruación; debe guardar silencio e irse de la casa a un lugar cercano, fácil de encontrar. Desde ese momento le está prohibido consumir alimentos cocidos, sal y toda clase de carne. Ella puede regresar a su casa por la noche pero no debe permitir que sus familiares le vean el rostro y debe acostarse en completo silencio.

La kókora es un gorro elaborado con hojas de rascadera, rascador o sámago. Se lo colocan a las muchachas cuando llegan a la pubertad. El gorro se hace de modo que quede una especie de pico en la parte superior, a manera de triángulo. El gorro cubre completamente la cabeza dejando solamente un orificio para ver y para la boca. La importancia de la imposición de la kókora radica en el hecho de que mediante ella se le da participación a la muchacha en la vida de los adultos, se le previene de enfermedades y se le augura buena fortuna durante su vida. Por esa razón se deben soplar

16. Inventario de Instituciones y Programas de Salud en las Fronteras Andinas, Op. cit.

17. Informe FNSPI (Foro Nacional de Salud de los Pueblos Indígenas de Costa Rica): Situación de las condiciones de salud de los pueblos indigenas en Costa Rica (2007). http://www.ministeriodesalud. go.cr/ops/saludpueblosindigenas/ (consultado el 10 de marzo de 2007). PARRA, A.: Situación de salud de las comunidades indígenas a orilla del rió Putumayo en el Departamento del Amazonas, Colombia, Secretaría Departamental de Salud del Amazonas, 1999. 
Mujer, salud y tradición en los U'wa de Colombia y cuestiones de género en las...

\section{Figura 2: Joven U'wa con la kókora}

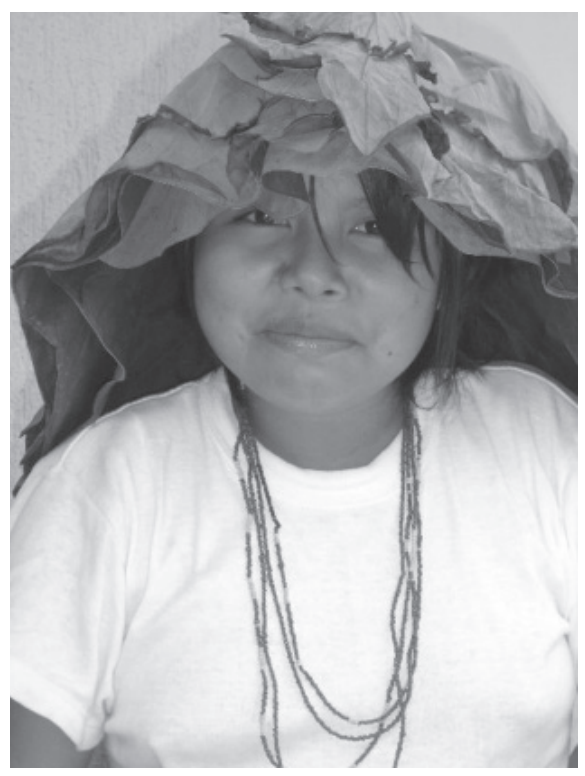

todos los objetos que ella va a utilizar, así como la comida asada como el maíz y el plátano verde, de tal forma que no vaya a sufrir de asma y enflaquecimiento» ${ }^{18}$.

Hoy en día se ha suavizado un poco y les deja la cara al descubierto, como muestra la fotografía.

Hay un interesante estudio del Dr. Alarcón-Nivia sobre consideraciones antropológicas y religiosas alrededor de la menstruación, tanto en culturas occidentales como en las comunidades indígenas. Cuando menciona los ritos de los pueblos U'wa, explica que:

"La tribu U'wa, de la familia Tunebo, que habita en la Sierra Nevada de Chita o Cocuy (alrededor del lago Bekana, al que consideran como el centro del mundo), impone a las jóvenes adolescentes de esta comunidad un sombrero llamado kókora, hecho con hojas de rascadera, el día que les llega la menstruación. El sombrero de kókora, que se lleva por lo menos un año, debe protegerla de los trastornos de la menstruación, del embarazo y ayudarla en la vida cotidiana. Durante el tiempo que la muchacha debe llevar la kókora deben observar normas muy estrictas. Entre éstas se encuentran el bañarse todos los días a las 4 de la mañana y no dejar ver su cabellera por nadie. Antiguamente el kókora se llevaba durante cuatro años. Actualmente solamente la llevan más o menos durante un año. Este rito también se llama 'rito de paso' y lo deben celebrar con la finalidad de prevenirle enfermedades a la muchacha en la segunda etapa de la vida y evitar que se presenten problemas durante las menstruaciones, el embarazo, el parto y la vida cotidiana.

18. Aguablanca, E. B.: Op. cit. 
Para quitar la kókora existe otra ceremonia: cuando una muchacha ya ha cumplido, según el jefe Uejená y según sus padres, con el tiempo previsto, se prepara una fiesta a la cual se invita mucha gente pues se ofrece un banquete con bastante carne de monte y plátano. Cuando le quitan la kókora su pelo debe aparecer largo y liso; su rostro debe aparecer rosado y elegante» ${ }^{19}$.

\section{CUESTIONES DE GÉNERO EN COMUNIDADES INDÍGENAS AMERICANAS}

Los estudios que incorporan la perspectiva de género a la comprensión del mosaico étnico-cultural que perfila a las sociedades indoamericanas son de gran actualidad:

«Las dinámicas impuestas por un lado, por el desmoronamiento del bloque socialista... y por otro, por la globalización... están contribuyendo a la redefinición de las identidades, proceso en el que está cobrando relevancia la etnicidad, la diferencia cultural.

Estos aspectos están siendo considerados hoy día en los análisis sociales, superando la postura que privilegiaba el estudio de las desigualdades económicas sin considerar otras determinantes en la dinámica social. Sin embargo, son escasos aún los estudios que incorporan la perspectiva de género a la comprensión del mosaico étnico-cultural que perfila a cada sociedad, no obstante que éste se vincula estrechamente con las relaciones de género, especialmente en sociedades donde los pueblos indígenas constituyen una mayoría de la población, y han ido adquiriendo relevancia en las últimas décadas» ${ }^{20}$.

Pero, por razones obvias, existen muchas más publicaciones que tratan de manera general sobre las comunidades indígenas de América del Norte que de Centro y Sur América, y muchísimas más en inglés que en español. Lo mismo puede decirse cuando nos referimos específicamente a cuestiones de género, que es un aspecto poco investigado aún en estas poblaciones. En una conocida base de datos exhaustiva sobre el tema de las comunidades indígenas, la Native American Studies Collections ${ }^{21}$, al apartado Women sólo se le dedica media página de 22, aunque, por ejemplo, se han estudiado aspectos muy concretos en indios norteamericanos como la homosexualidad y el "tercer género», o personas con características masculino-femeninas ${ }^{22}$. Este nivel de detalle en investigaciones

19. AlarCón-Nivia, Miguel Ángel: "Algunas consideraciones antropológicas y religiosas alrededor de la menstruación", Revista Colombiana de Obstetricia y Ginecologia 56 (2005), pp. 1-15. (consultado http://www.scielo.org.co/scielo.php?script=sci_arttext\&pid=S0034-74342005000100005$\& \operatorname{lng}=e s \& n r m=i s o$ el 12 de marzo de 2007).

20. MONZÓN, Ana Silvia: «La diversidad es riqueza: las relaciones de género en sociedades pluriculturales. Propuestas para la reflexión", Serie cuadernos de trabajo hacia la equidad, Unión Mundial para la Naturaleza. Fundación Guatemala, 2003 (consultado el 10 de noviembre de 2006 en http://www.generoyambiente.org/admin/admin_biblioteca/documentos/diveridad\%20riqueza.pdf).

21. Native American Studies Collections, consultado en http://library.stanford.edu/depts/ssrg/native/ nativepm.html el 18 de noviembre de 2007.

22. Roscoe, Will: The Zuni Man-Woman, Albuquerque, University of New Mexico Press, 1991. 
Mujer, salud y tradición en los U'wa de Colombia y cuestiones de género en las...

específicas está ausente en estudios de comunidades nativas centro y sudamericanas.

En términos generales, en las comunidades U'wa se reproducen muchos de los estereotipos que encontramos en otras culturas:

«Oficios del hombre: El hombre debía preparar el terreno para la siembra, rozar y tumbar los palos, repicar la tierra o quemar, y en ocasiones colaborar en la siembra. También se debía dedicar a buscar la leña, conseguir la carne de caza, preparar el caraño, la otoba y quemar las ollas recién hechas; asimismo, estaba encargado de construir los ranchos o chozas, organizar las fiestas familiares y salir a intercambiar los productos a otros sitios donde debía conseguir sal y otras cosas que faltaran en el hogar. En algunas comunidades de la región de Aguablanca los hombres son los encargados de desyerbar las huertas porque a las mujeres solamente se les asignan los oficios domésticos y de crianza de los hijos.

Oficios de la mujer: A la mujer le estaban asignadas todas las actividades de crianza de los hijos además de los oficios domésticos como cocinar los alimentos, preparar la chicha, recoger los productos de la huerta para preparar las comidas, buscar las hojas para preparar las camas, elaborar las ollas, las mochilas y trampas para cazar aves y otros animales. En algunas comunidades de esta región las mujeres desyerban las huertas mientras que en otras son los hombres quienes lo hacen ${ }^{23}$.

Así, las conocidas desigualdades de género que se dan en muchos países sudamericanos (por ejemplo, en índices de analfabetismo) se ven incrementadas, siempre en contra de las mujeres, en las comunidades indígenas. El presente estudio lo confirmó, con relación a la comunidad U'wa. En otros países de la región, como Panamá, la situación de la mujer indígena es similar. Afortunadamente, hay iniciativas de investigación en la acción y de abordaje de cuestiones básicas desde la perspectiva de género (tanto en la masculinidad como en la feminidad $)^{24}$. De hecho, una de las líneas prioritarias de actuación en los programas internacionales es el tratamiento de «la condición de las mujeres en los aspectos educativos, de salud, de autonomía económica, de participación y violencia de género ${ }^{25}$. Las mujeres U'wa de nuestro estudio enfrentan, como otras del continente americano, una "triple desventaja debido a su a) pertenencia étnica, b) sexo y c) residencia predominantemente rural» ${ }^{26}$.

23. Aguablanca, E. B.: Op. Cit.

24. Véase, por ejemplo, el proyecto panameño "Género y desarrollo", dirigido a las comunidades indígenas. (consultado en http://www.ceaspa.org.pa/index.php?option=com_content\&task= view\&id=14\&Itemid=1 el 12 de noviembre de 2007).

25. AECI, Agencia Española de Cooperación Internacional: Plan de Acción para la Equidad de Género - Diagnóstico y Plan Operativo 2007-2008, Plan de Acción Regional. (consultado el 1 de octubre de 2007 en http://ceccsica.org/programas-accion/genero/planregional2.html\# (consultado el 19/XI/07).

26. "Género, equidad y la salud de las mujeres indígenas en las Américas", Hoja informativa del programa Mujer, Salud y desarrollo, de la Organización Panamericana de la Salud. (consultado el 25 de octubre de 2007 en http:/www.ops-oms.org/Spanish/DPM/GPP/GH/ GEHFactSheetSpanish.pdf) 
Concluiremos citando un interesante estudio ${ }^{27}$ donde se presentan reflexiones y conceptos básicos para comprender la diversidad étnica y su vínculo con las relaciones de género, pues ambas dimensiones se entrecruzan e influyen mutuamente. Se analizan también las brechas sociales entre mujeres y hombres indígenas, sustentando la idea de que las relaciones de género al interior de los pueblos indígenas, si bien están matizadas por cosmovisiones y prácticas propias, están estructuradas por la cultura patriarcal que subordina y discrimina a las mujeres como género. Algunos movimientos indígenas han planteado reivindicaciones que se han plasmado en normativas legales -nacionales e internacionales- que han llegado a conformar un campo particular de derechos de los pueblos indígenas. En este punto tienen relevancia especial las luchas específicas de las mujeres indígenas. El estudio analiza también elementos básicos de las corrientes de pensamiento que actualmente tratan de explicar y proponer ideas y acciones en el ámbito de las relaciones interétnicas en sociedades multiculturales, pluriétnicas y plurilingües. Aunque estos estudios se han dirigido inicialmente a países centro y sudamericanos, los flujos migratorios actuales a nivel mundial los convierten en referencias de interés para las latitudes europeas. Concluiremos con una cita, en este contexto:

«Las mujeres comparten la opresión étnica con los hombres de sus grupos, por el sólo hecho de ser parte de pueblos tratados como minorías bajo dominación. Sin embargo, esta opresión es diferente de la que viven los hombres porque ellas están sujetas, además, al dominio genérico de los hombres de las familias -y quienes desempeñan cargos de autoridad- de las comunidades a las que pertenecen. En este sentido, las etnias son modificadores que dan una especificidad a la condición de la mujer $\aleph^{28}$.

27. MonZón, Ana Silvia: Op. Cit.

28. Ibíd. 